\title{
SPECIAL ASPECTS OF EVALUATING THE CRACKING RESISTANCE OF BEAMS WITH PRESTRESSED REINFORCEMENT IN THE PROGRAM OF THEIR BENCH TESTS
}

\author{
Alexander Sim*, Igor Belutsky, \\ Pacific National University, Russian Federation
}

This article provides information on the "Bypass of Khabarovsk city" road and all its associated transport infrastructure facilities. A method for evaluating the crack resistance of beams under bench test conditions is given. Based on the provisions of the theory of bending, expressions are presented for determining stresses for crack resistance.

Key words: prestressed concrete beam, testing on the ground, crack resistance, relative deformation, stress, bending of the beam

\section{INTRODUCTION}

Increasing the crack resistance of reinforced concrete structures is an urgent task of the present time. This is confirmed by research conducted in various universities around the world $[1,2,3,4,5,6]$.

In 2017, the construction of a large promising facility - the "Bypass of Khabarovsk city» road-began in the far East of Russia. The project will relieve the city roads of Khabarovsk, create a single transport corridor Chita-Vladivostok, as well as provide an increase in freight and passenger traffic with the countries of North-East Asia [7].

The project includes the construction of not only the road itself, but also all related transport infrastructure facilities. The route will pass through 5 new transport interchanges, 24 bridge structures. The total length of the high-way will be 26.8 kilometers [8].

For the construction of bridge crossings and overpasses of the route "Bypassing Khabarovsk city" , non-standard beams for the Far East are used. In Khabarovsk, the production of these reinforced concrete beams rein-forced with tensioned reinforcement is deployed. After production of beams by the staff of Department "Highways" of the Pacific National University bench tests were carried out.

One of the stages of the bench test program for beams of bridge structures of the road "Bypass of Khaba-rovsk city" included an assessment of the compliance of span structures with the requirements relating to the conditions of serviceability limit states. The beam test was done according to the scheme, which included loading the beam with a vertical load [9]. The value of the test load is determined from the considerations that the force effect of the test load forms in the tested beam a stress state similar to those that occurs in a real span by serviceability limit-state design.

The bench test program included testing beams with a length of $12,15,18,21,24,33 \mathrm{~m}$. The Stroyproekt
In-stitute developed the design documentation for the span structures of the specified length that contained data necessary to characterize the force effects and the stress state that are adequate to the actual working conditions of the load-bearing structures in the span structures. Thus, under the test load, forming a positive bending moment in normal cross sections of the beam, the strain gauges attached to the upper flange and its lower flange (Fig. 2) could record relative tension strain $\varepsilon_{b}$ in the upper flange and compression strain $\varepsilon_{H}$ in the fibers of the lower flange. It is quite clear that the relative strains $\varepsilon_{H}$ could not be comprehensive information in the characteristic of the stress state of the lower flange of the beam, much less of its crack resistance, because only the effect of the vertical test load predetermining the positive bending moment was recorded, and they did not carry information about the effect of the prestressing forces, in essence, creating the resource of crack resistance of the concrete of the lower zone and realizing the effect of prestressing in reinforced concrete structures of bridge span structures.

In this regard it is worth noting that at the moment of prestressing the concrete of the beam, the conditions of the bench tests did not allow the strain gauges to be placed on the lower flange of the beam covered with the formwork, the window arrangement of which could make it inoperable and was not planned in the bench test program.

\section{METHOD OF ESTIMATION OF CRACK RESISTANCE OF REINFORCED CONCRETE BEAMS}

To build an algorithm for finding the relative strain of interest $\varepsilon_{d^{\prime}}, \varepsilon_{u}$ that are unmeasurable at the moment the prestressing force is transferred to the concrete of the beam, we consider a diagram reflecting the characteristic pic-ture of the strained condition of the beam from the effect of prestressing forces, fig. 1.

For example, the bending line of a beam A.B.C. is described by a circular curve. We take into account that the angle supported on the diameter of the circular curve 


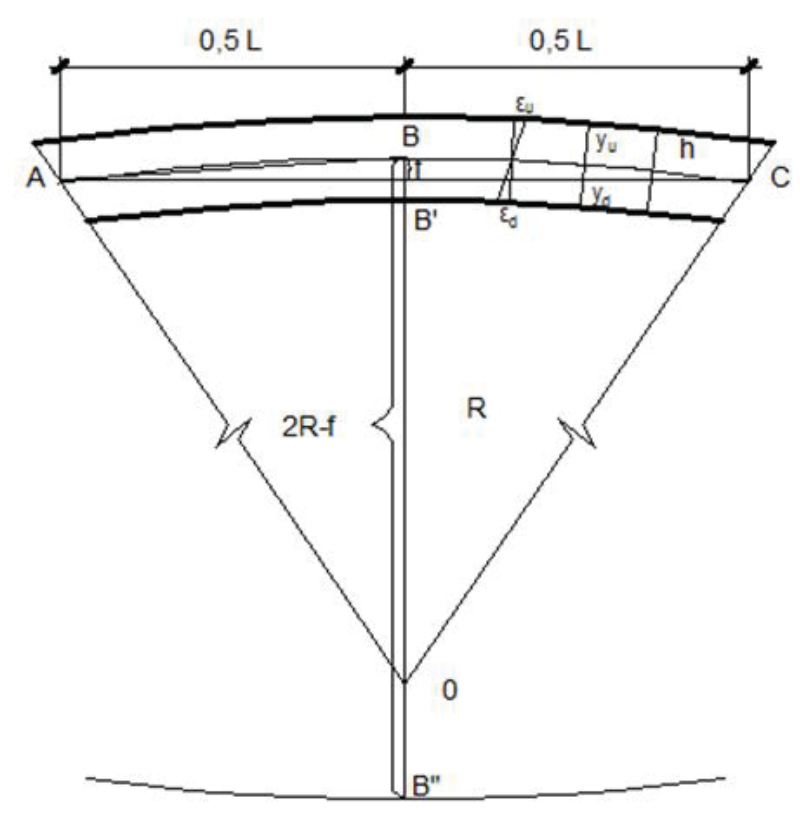

Figure 1

B.A.B" is right, and the height A.B' in the right-angled triangle $B A B "$, dropped from the right angle $A$ to the hypotenuse BB", divides it into segments $f=\mathrm{B}^{\prime}$; and B. $\mathrm{B}^{\prime \prime}=2 R-f$, which, taking into account the notation in fig. 1, follow the dependence:

$\frac{f}{0,5 L}=\frac{0,5 L}{2 R-f}$

from which the radius of the circular curve of the beam follows

$R=\frac{0,25 L^{2}+f^{2}}{2 f}$

bending $f$ from the prestressing forces.

Having found the radius of curvature R, we use the relationship known from the bending theory

$\frac{1}{R}=\frac{M}{E I}$

Expanding in the right part of the expression (3) its components in the form

$$
\begin{gathered}
\frac{M y_{d}}{I_{\text {bred }} y_{d}} \\
\frac{M y_{u}}{E I_{\text {bred }} y_{u}}
\end{gathered}
$$

we get the relations

$\frac{1}{R}=\frac{\varepsilon_{d}}{y_{d}}$ and $\frac{1}{R}=\frac{\varepsilon_{u}}{y_{u}}$

Using substitution $y_{d}=h-y_{u}$ we get

$\frac{1}{R}=\frac{\varepsilon_{d}}{h-y_{u}}=\frac{\varepsilon_{u}}{y_{u}} ; \quad \varepsilon_{d}=\frac{y}{R} ; \quad \varepsilon_{u}=\frac{y_{u}}{R} ;$

$\frac{h-y_{u}}{\varepsilon_{d}}=R$ and $\frac{y_{u}}{\varepsilon_{u}}=R$ and after transformations we find

$y_{u}=\frac{h \cdot \varepsilon_{u}}{\varepsilon_{d}+\varepsilon_{u}}, y_{d}=\frac{h \cdot \varepsilon_{d}}{\varepsilon_{d}+\varepsilon_{u}}$

Thus, with the known values of the concrete modulus of elasticity, taking into account the found relative strains $\varepsilon_{d}$, and $\varepsilon_{u}$ by the formula (7), it is possible to evaluate the stress state of the normal section of the beam at the extreme lower and upper fibers, respectively

$\sigma_{d}=\sigma_{d} E_{b c}$,

$\sigma_{u}=\varepsilon_{u} E_{b p}$

where $E_{b c}, E_{b p}$ are the values of the concrete moduli of elasticity when working in compression and tension, respec-tively.

Taking into account the previously agreed situation with the conditions of bench tests relating to the impossi-bility of measuring the relative strains $\varepsilon_{d}$, $\varepsilon_{u}$ during the transfer of prestressing forces to the concrete of the beam, the stresses $\sigma_{d}, \sigma_{u}$ found by the formulas (9), (10) characterize the effect of prestressing forces. But for an com-prehensive characteristic of the compliance of the fabricated structure to the crack resistance requirements in accor-dance with the serviceability limit states of the span structures of road bridges, it is necessary to take into account the stresses from the test load, the value of which was found because the test load force forms with the efforts of the prestressing the stress state similar to those that occurs in the beam of a real span by serviceability limit-state design.

In this connection, we give an estimate of the stress state of the beam, cross section of which is shown in Fig. 2, having accepted the principle of superposition with regard to the sequence of formation of the stress state. At the stage of transfer of prestressing forces $N_{0}$ to the concrete of the beam the stresses in the lower and upper fibers of the beam will be equal, respectively, to

$$
\begin{gathered}
\sigma_{b H}=-\frac{N_{o}}{A_{\text {bred }}}-\frac{N_{p} y_{p} y_{d}}{I_{\text {bred }}} \\
\sigma_{b s}=-\frac{N_{p}}{A_{\text {bred }}}+\frac{N_{p} y_{p} y_{u}}{I_{\text {bred }}}
\end{gathered}
$$

At the stage of loading the beam with a test load, creating a bending moment Mtest in a controlled section, stresses occur in the lower and upper fibers of the beam, which can be found from the following expressions respec-tively

$\sigma_{b d}=+\frac{M_{\text {test }}}{I_{\text {bred }}}$

$\sigma_{b u}=-\frac{M_{\text {test }} y_{u}}{I_{\text {bred }}}$

Thus, the compliance of the fabricated structure with the requirements of the serviceability limit states relat-ing to crack resistance will be ensured if 


$$
\sigma_{b c}=+\frac{M_{\text {test }} y_{d}}{I_{\text {bred }}}-\frac{N_{P}}{I_{\text {bred }}}-\frac{N_{p} y_{p} y_{d}}{I_{\text {bred }}}<2 R_{b t s e r}
$$

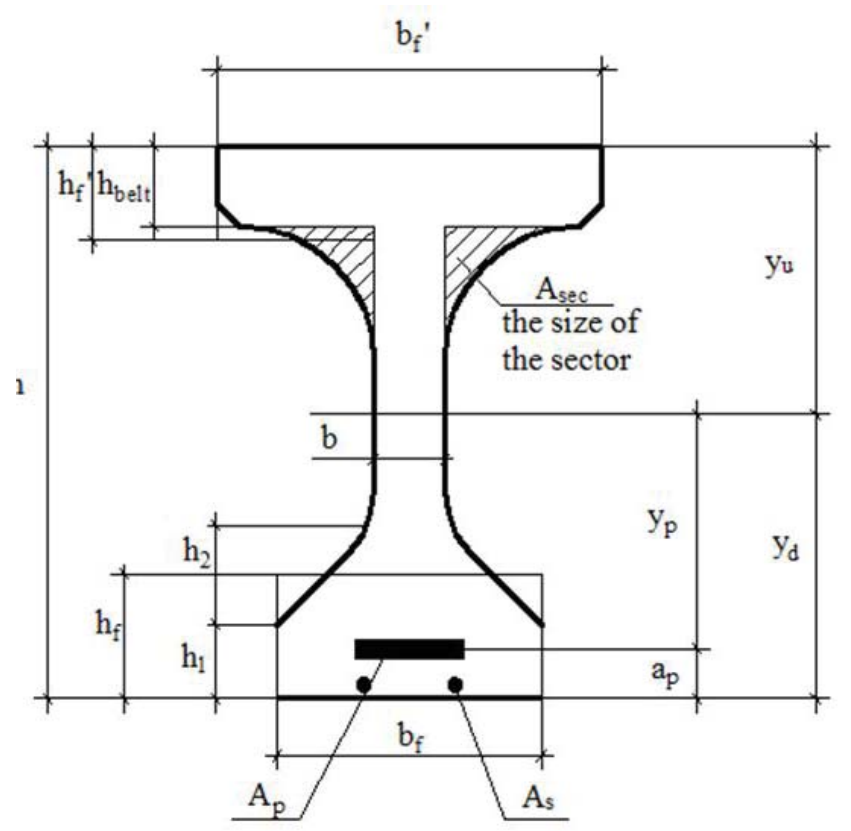

Figure 2

The right part of expression (15), as the restrictive part of the crack resistance condition, is adopted in clause 7. 96 of Code of Practice 35.13330.2011 (Ministry of regional development of the Russian Federation) and corres-ponds to the variant of mixed reinforcement of the lower zone of beam adopted in the design decisions of the de-signer of the Stroyproject Institute for the supporting structures of bridge structures on the road "Bypass of Khaba-rovsk city". Thus, the block of expressions (11), (12), (13), (14) forms the basis for estimating the stress state of the beam in accordance with the sequence of force effects on the beam: expressions (11), (12) reflect the action of prestressing forces; expressions (13), (14) characterize the effect of the test load, and their combination in the form of (15) gives an assessment of the compliance of the fabricated beam with the requirements of the serviceability limit states. It should be noted that expressions (11)-(14) are deduced based on the traditional view of the behavior of reinforced concrete, based on which concrete is traditionally considered a homogeneous isotropic material, working with a modulus of elasticity in tension equal to the modulus of elasticity in compression. At the same time, the paper [10] points out objective reasons for the difference in the elastic moduli of a concrete when working in compression and tension and the validity of considering concrete a bimodular material in the behavior of a bending element $[11,12]$, the compressed zone of which works with modulus of elasticity in compression, and stretched zone with modulus of elasticity in tension. Earlier, expressions (9), (10) reflected the differentiated approach to the evaluation of stresses of compressed and stretched zones, taking into account the elastic moduli characterizing the operation of these zones. As an alternative, block of expressions (11)-(14) were suggested as solutions. But in this case, the fact is important that the geometric characteristics, in particular, the reduced moment of inertia $I_{\text {bred }}$ in expressions (11), (12) is different in comparison with the moment of inertia in expressions (13), (14) both in quantitative terms and semantic content.

As the block of expressions (11), (12) and the block of expressions (13), (14) characterize the effect of bend-ing moments of different sign on the beam, when it is quite likely that the concrete is bimodular in the bending structure. Indeed, if at the prestressing stage, the concrete of the lower zone of the beam compresses and works with the modulus of elasticity in compression, then at the next test stage, when the beam is loaded with a vertical test load, the zones of compressed and stretched concrete are changed. In other words, the design model changes in this situation, the principle of superposition is unfair, and the evaluation of the stress state using expressions in the form (11), (12), (13), (14) and moments of inertia regardless of the direction of the bending moment acting in the section of the bending element at different stages of loading with the subsequent summation of the results, as is the case in expression (15), is incorrect. In this regard, a different approach to the evaluation of the stress state is suggested, which consists in choosing a working scheme in the terminology of I. M. Rabinovich [13]. In this case, the term "working scheme" means such a stress diagram that would adequately reflect the total of the effect of all loads preceding the test phase concerned, adopted for a specific section of the bench test program as a control position in assessing the conformity of the manufactured design to the project requirements.

It is suggested to evaluate the stress state of the cross section of beam with the determination of the stresses of interest in the lower zone of the beam, which represent the main object of attention in assessing the conformity of the manufactured beam to the requirements of the serviceability limit states relating to cracking, by consideration of the normal stress diagram in the cross-section of beam, fig. 3 .

Obviously, to start the procedure of solving the problem in the stated format, it is necessary to specify zones of compressed and stretched concrete. Perhaps, this situation can be brought into certainty by taking into account that tests aimed at assessing the conformity of fabricated structures with the project requirements concerning the conditions of the serviceability limit states are predominant. The limitation of tensile stresses in the lower zone of the beam is equal to $2 R_{b t s e r}$, which means that the tensile stresses in the lower zone of the beam and the operation of concrete in this zone with modulus of elasticity in tension $E_{b p}$ are most likely at the final stage of the test, what, in fact, the final result of expression (15) indicates. It reflects in principle the totality of effect of loads included in the calculation of spans with prestressed reinforcement in order to assess their compliance with the requirements of crack resistance. 


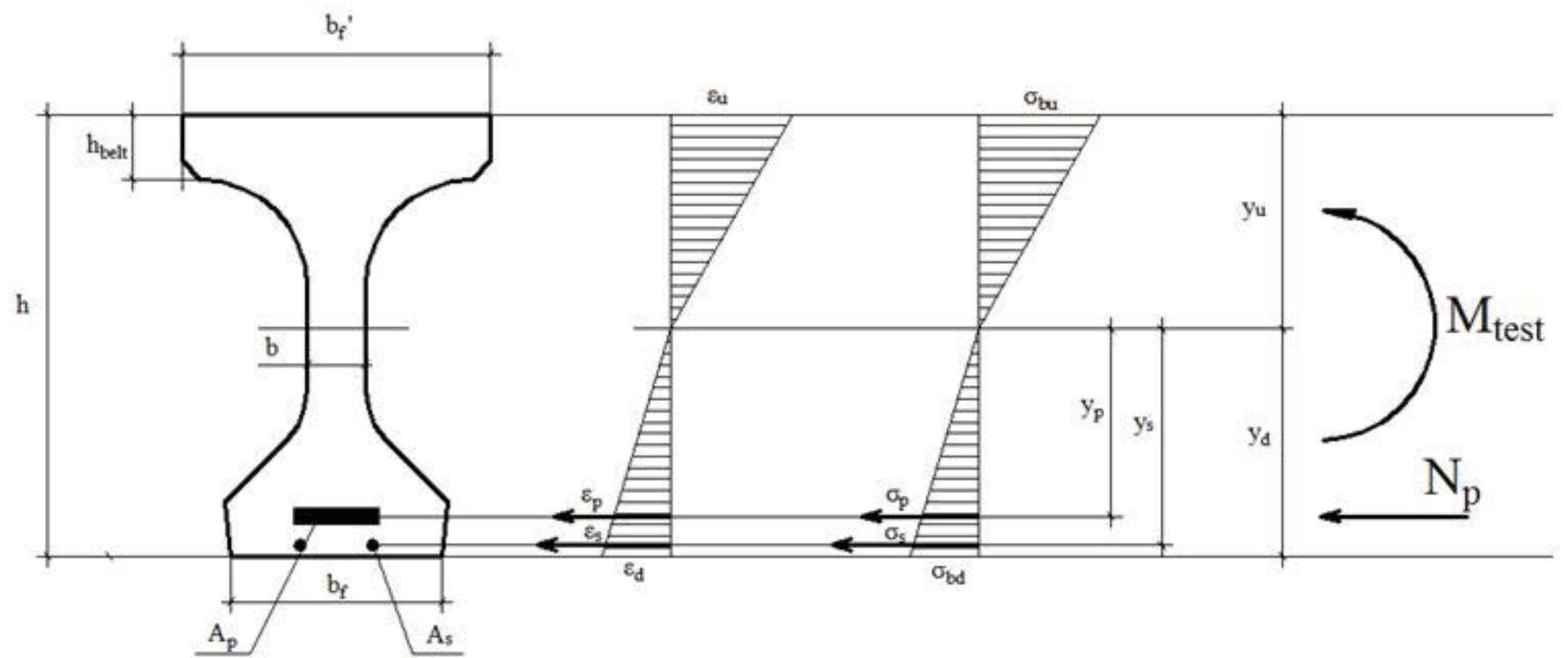

Figure 3

Considering the stress-strain state of the section in fig. 2 we will bring its geometry to the traditional form with the reduced thickness of the compressed flange and the lower flange, respectively (Fig. 2)

$h_{s}^{\prime}=h_{\text {belt }}+\frac{2 A_{\mathrm{sec}}}{b_{f}^{\prime}-b} ; h_{\varphi}^{\prime}=h_{1}+\frac{h_{2}}{2 b_{f}}\left(b_{f}-b\right)$.

Take fair the law of plane sections with respect to the change in relative strains over the entire interval of change $y_{i}$ from $y_{d}$ to $y_{u}$; take linear the law of stress variation within each of the zones (Hooke's law) when concrete works with moduli of elasticity in tension $E_{b p}$ in the lower zone, and with moduli of elasticity in compression $E_{u c}$ in the upper zone. With the fairness of the law of plane sections, the following relations are correct

$\frac{\sigma_{b u}}{E_{b c} y_{u}}=\frac{\sigma_{b d}}{E_{b p} y_{d}}=>\sigma_{b u}=\frac{\sigma_{b d} E_{b c} y_{u}}{E_{b p} y_{d}}$

The condition of compatibility of strains of concrete and reinforcement along the line of their contact gives grounds for the following expression

$$
\frac{\sigma_{s}}{E_{s} y_{s}}=\frac{\sigma_{b d}}{E_{b p} y_{d}}=>\sigma_{s} \frac{\sigma_{b d} E_{s} y_{s}}{E_{b p} y_{d}}
$$

Determine the forces along the beam axis in its upper zone

$N_{b}=\frac{1}{2} \sigma_{b u} b y_{u}+\sigma_{b u} h_{f}^{\prime}\left(b_{f}^{\prime}-b\right)$

in its lower zone

$$
N_{d}=\frac{1}{2} \sigma_{b d} b y_{d}+\sigma_{b d} h_{f}\left(b_{f}-b\right)+\sigma_{s} A_{s}-N_{p} \text {. }
$$

Then the equilibrium condition, taking into account the different directions of efforts in the upper and lower zones (Fig. 3), can be represented

$N_{u}-N_{d}=0$

Developing $N_{u}$ by formula (19), $N_{d}$ by formula (20), we get

$\frac{1}{2} \sigma_{b u} b y_{u}+\sigma_{b u} h_{f}^{\prime}\left(b_{f}^{\prime}-b\right)-\frac{1}{2} \sigma_{b d} b y_{d}+$

$+\sigma_{b d} h_{f}\left(b_{f}-b\right)-\sigma_{s} A_{s}-N_{p}=0$.

and

$$
\begin{aligned}
& \sigma_{b u}\left[\frac{1}{2} b y_{u}+h_{f}^{\prime}\left(b_{f}^{\prime}-b\right)\right]-\sigma_{b d}\left[\frac{1}{2} b y_{d}+h_{f}\left(b_{f}-b\right)\right]+ \\
& +N_{p}-\sigma_{s} A_{s}=0 .
\end{aligned}
$$

After replacing by formula (17), and by formula (18) we have

$$
\begin{aligned}
& \sigma_{b d} \frac{E_{b c} y_{u}}{E_{b p} y_{d}}\left[\frac{1}{2} b y_{u}+h_{f}^{\prime}\left(b_{f}^{\prime}-b\right)\right]-\left[\frac{1}{2} b y_{d}+h_{f}\left(b_{f}-b\right)\right]- \\
& -\frac{\sigma_{b d} E_{s} y_{s}}{E_{b p} y_{d}} A_{s}=N_{p} .
\end{aligned}
$$

grouping the similar and making the substitution,

$$
y_{d}=h-y_{u} ; y_{s}=y_{d}-a_{s} \text {. }
$$

we get

$\sigma_{b d}\left\{\begin{array}{l}E_{b c} y_{u}\left[\frac{1}{2} b y_{u}+h_{f}^{\prime}\left(b_{f}^{\prime}-b\right)\right]-\frac{1}{2} b y_{d}+ \\ +h_{f}\left(b_{f}-b\right)-E_{s} y_{s} A_{s}\end{array}\right\}=N_{p} E_{b p} y_{d}$,

from which it follows

$$
\sigma_{b d}=\frac{N_{p} E_{b p}\left(h-y_{u}\right)}{\left\{E_{b c} y_{u}\left[\frac{1}{2} b y_{u}+h_{f}^{\prime}\left(b_{f}^{\prime}-b\right)-\left(\frac{1}{2} b y_{d}+h_{f}\left(b_{f}-b\right)\right)\right]-E_{s} A_{s}\left(h-y_{u}-a_{c}\right)\right\}} .
$$


Analysis of expression (27) shows that if we have data on the prestressing force $N_{p}$, the cross-sectional geometry of the beam, found by (16), the reduced thickness of the upper flange $h_{f}^{\prime}$ and the lower flange $h_{f}$ of a given area of bar reinforcement in the lower zone of the beam $A_{s}$, its modulus of elasticity $E_{s}$ by variations of the values $y_{u}, y_{d}$ with the obligatory observance of the condition $y_{u}+y_{d}=h$, where $h$ is the height of the beam from the geometry of its cross section, we can find the stresses $\sigma_{b d}, \sigma_{b u}$ by the formula (11), $\sigma_{s}$ by the formula (18), which would satisfy the condition of equilibrium $\Sigma x=0$ according to (22).

At the same time, the expression (27) clearly shows the dependence of stresses $\sigma_{b d}$ on the value of the mod-ulus of elasticity in tension of concrete $E_{b p}$, which is quite natural, because the model $\sigma_{b d}$ is based on considering the pattern of relative strains of the longitudinal fibers of the beam as a function of efforts that adequately reflect the totality of design loads determining actual the conditions of the serviceability limit states in the operation stage, and checking the security of the specified conditions in the fabricated beam design at the stage of the beam test.

The value of the modulus of elasticity in tension, work of which is most likely in the lower zone of the beam at this stage of bench tests under the action of prestressing forces $N_{p}$ and vertical test load, which determines the bending moment $M_{\text {test }}$ (Fig. 3). It is suggested to evaluate the modulus of elasticity $E_{b p}$ through the solution of the problem, which is based on the equality of moments of external and internal forces.

When solving a problem:

1. the initial data is the cross-sectional geometry of the beam (Fig. 2), the area and modulus of elasticity of bar reinforcement in the lower flange of the beam $A_{s}$ $E_{s}$, creating the effect of mixed reinforcement, the area and modulus of elasticity of prestressed reinforcement $A_{s} E_{p}$, their position relative to the neutral axis, respectively $y_{s,} y_{p}$;

2. the working hypotheses are the following the true hypothesis of plane sections regarding the nature of the distribution of relative strains along the height of the beam section, and true condition of the compatibility of strains of concrete and reinforcement along the line of their contact.

The truth of the hypothesis of plane sections gives reason for the equality of relations (Fig. 3)

$\frac{\varepsilon_{u}}{y_{u}}=\frac{\varepsilon_{d}}{y_{d}}$

which allow us to find the position of the neutral axis from the expressions

$y_{d}=\frac{h \varepsilon_{d}}{\varepsilon_{u}+\varepsilon_{d}} ; \quad y_{u}=\frac{\varepsilon_{u} h}{\varepsilon_{u}+\varepsilon_{d}}$

The validity of the expressions is confirmed by the derivation of expressions (8).

The true hypothesis of plane sections and condition of compatibility of strains of concrete and reinforcement allow to write

$$
\frac{\varepsilon_{u}}{y_{u}}=\frac{\sigma_{s}}{E_{s} y_{s}}=\frac{\sigma_{p}}{E_{p} y_{p}}
$$

and get stresses $\varepsilon_{p}, \sigma_{p}$ for bar and prestressed reinforcement.

$\sigma_{s}=\frac{\varepsilon_{u} E_{s} y_{s}}{y_{u}}$

$\sigma_{p}=\frac{\varepsilon_{u} y_{p} E_{p}}{y_{u}}$

characterizing the stress state of the reinforcement in the structure of the reinforced concrete element at the end of the current test stage, after the prestressing forces are transferred to the concrete of beam and the effect of last in-crement of the test load.

Thus, considering reliable the data on the beam tests relating to measured strains, estimated concrete defor-mability, represented by its modulus of elasticity in compression as more available information, and also taking into account the values of $y_{d}, y_{u}$ found by the formula (29) and $\sigma_{s,} \sigma_{p}$ found by the formula (31), (32). The resisting moment has a particular value:

moment of force of the upper zone of the beam relative to the neutral axis

$M_{\text {вз }}=\frac{1}{2} \sigma_{b u} b y_{u} \frac{2}{3} y_{u}+\sigma_{b u} h_{f}^{\prime}\left(b_{f}^{\prime}-b\right)\left(y_{u}-0,5 h_{f}^{\prime}\right)$.

In the expression (33), the fiber stresses $\sigma_{b u}$ correspond to the dependence $\sigma_{b u}=\varepsilon_{u} E_{b c}$; the reduced thickness of the upper flange $h_{f}^{\prime}$ and the lower flange are found by the formula (16).

The moment of force of the lower zone of the beam will be represented as follows

$$
\begin{aligned}
& M_{\text {нз }}=\sigma_{s} A_{s} y_{s}+\sigma_{p} A_{p} y_{p}+\frac{1}{2} \varepsilon_{d} E_{b p} b y_{d} \cdot \frac{2}{3} y_{d} \\
& +\varepsilon_{d} E_{b p} b_{f} h_{f}\left(y_{d}-0,5 h_{f}\right)
\end{aligned}
$$

and more compactly

$$
\begin{aligned}
& M_{н з}=\sigma_{s} A_{s} y_{s}+\sigma_{p} A_{p} y_{p}+\frac{1}{2} \varepsilon_{d} E_{b p} b y_{d} \cdot \frac{2}{3} y_{d} \\
& +\varepsilon_{d} E_{b p} b_{f} h_{f}\left(y_{d}-0,5 h_{f}\right)
\end{aligned}
$$

Then, from the equality of the moment of internal forces (as the sum of expressions (33) and (34)) and the moment of external forces generated by the test load $M_{\text {test }}$ and the prestressing forces $N_{p} \cdot y_{p}$ (Fig. 3), taking into account their different directions equal to $M_{\text {load }}=M_{\text {tast }}-N_{p} \cdot y_{p}$ it is possible to find the value of the modulus of elasticity in tension $E_{b p}$.

$E_{b p}=\frac{M_{t e s t}-N_{p} y_{p}-\sigma_{s} A_{s} y_{s}-\sigma_{p} A_{p} y_{p}}{\varepsilon_{d}\left(\frac{1}{3} b y_{d}^{2}+b_{f} h_{f}\left(y_{d}-0,5 h_{f}\right)\right.}$ 
After finding the modulus of elasticity in tension by the formula (35), the stresses in the lower zone of the beam are found by (27), and in the upper zone of the beam by (17), the stresses in the bar reinforcement by (18). The stresses in the prestressed reinforcement corresponding to the work of the beam at the final stage of this stage of testing with the validity of the hypothesis of plane sections and the conditions of compatibility of strains of concrete and reinforcement are equal to

$$
\sigma_{p}=\frac{\sigma_{b d} E_{p} y_{p}}{E_{b p} y_{d}} .
$$

\section{CONCLUSIONS}

In our opinion, the suggested algorithm for evaluating the stress state of a reinforced concrete beam with prestressed reinforcement as part of a bench test program for a beam aimed to assess its compliance with the require-ments of the serviceability limit states certainly deserves attention and development for the following reasons:

- firstly, when building an algorithm of achieving a goal attention is drawn to the impossibility of measuring the relative strains in the beam at the stage of creating prestressing in it. In this regard, the expression (27) compen-sates for the lack of direct information on the fiber strains of the beam;

- secondly, the presented method of evaluating the stress state of a beam at the stage of its bench test is a single block of expressions that take into account the likely behavior of concrete in compression and tension with different moduli of elasticity, which required the selection and justification of the working scheme, which equally adequately reflects the behavior of the beam at the stage of transfer of prestressing forces to the concrete of the beam and at the stage of loading the beam with a vertical test load.

It is worth noting that the comparison of the results obtained by the suggested method with the results based on the expressions of the form (11), (12), (13), (14), (15) can assess the significance, the need to consider the beha-vior of concrete as bimodular structure in an objective assessment of the stress state of a reinforced concrete struc-ture, which may be the most significant in the considered problem in its use in other composite structures for which the history and loading sequence touch the principle of superposition and the principle of additivity.

\section{REFERENCES}

1. Berdichevskii GI and Borisova VA, 1969. Crack resistance of prestressed T-beams with wire reinforce-ment in diagonal bending. Beton i Zhelezobeton, (8), pp. 41-44. Retrieved from https://www.scopus. $\mathrm{com} /$ inward/record.uri?eid=2s2.00014554017\&partnerID $=40 \&$ md5=8cdb6a3c550d503bb95c90647bde61c.

2. Sharipov, R.S., 1988. Cracking resistance of the end sections of prestressed structures during the transmis-sion of compressive forces. Beton i Zhelezobeton, (6), pp. 14-16. Retrieved from https://www.scopus.com/inward/record. uri? eid $=2$ s2.00024028384 \& partner $I D=40 \&$ md5=d5adc489818ea22abfa0a92f6ec29513.

3. Kwak H.-G., Seo Y- J (2002) Numerical analysis of time-dependent behavior of precast prestressed con-crete girder bridges. Construction and Building Materials, 16 (1), pp. 49-63. DOI: 10.1016/S09500618(01)00027-7

4. Jiang, Q., Ye, X., Zhang, Y., Chong, X., Huang, X., Liu, G. (2014) Experimental research on cracking re-sistance of prestressed high-strength reinforced concrete beams. Jianzhu Jiegou Xuebao/Journal of Building Struc-tures, vol. 35, Issue 12, pp. 51-57, DOI: 10.14006/j.jzjgxb.2014.12.007.

5. Hu, Z., Du, R., Sun, L.Z., Wang, S., Wu, F., Xian, D. and YUAN, S., 2017. Experimental investigation of post-cracked flexural behavior for PC girder bridges, IABSE Conference, Vancouver 2017: Engineering the Future - Report 2017, pp. 64-75. DOI: 10.14006/j. jzjgxb.2014.12.007.

6. Toniolo, G, di Prisco, M. (2017) Bending moment. Toniolo, G, Springer Tracts in Civil Engineering. Springer, Milan, Italy. pp. 169-262. DOI: 10.1007/9783-319-52033-9_3.

7. Yarmolinsky V. A., Kondratenko T. E. (2018) Prospects for the development of toll roads in the Russian Far East. Transport construction. Issue № 08/2018, ISSN 0131-4300 pp. 8 - 11.

8. Khabarovsk Krai government. The official start of the construction of the highway "Bypass of Khabarovsk city», from https://khabkrai.ru/events/news/166328/, accessed on 2019-07-09. 
9. Belutskii I.Y., Sim A.D., Lapin A.V. (2018) Features of stress strain of beams with stressed reinfocement in the format of bench test. Far East: problems of development of architectural-construction and road-transport com-plex: materials of the International scientific-practical conference. - Khabarovsk: publishing House of Pacific na-tional University, vol. 2. issue, ISBN 978-5-7389-2735-5, pp. 214-219

10. 1Volkov G. F.(1962) The role of the concrete structure and ferrocement. «Ferrocement and ferrocement constructions». Gosstroyizdat, Moscow.

11. Katskhazov A. P.(1947) Road bridges. Dorizdat, Moscow.
12. Belutskii I.Y., Sim A.D. (2018) Concrete bi-modulus analysis in the estimation of stress state of the normal sections of reinforced bending element. IOP Conference Series: Materials Science and Engineering 463 (042070), pp. 1-5. DOl:10.1088/1757899X/463/4/042070

13. Rabinovich I. M. (1950) Construction mechanics course. Part 1. State publishing house of construction li-terature. Moscow. 